\title{
On q-integrals over order polytopes (extended abstract)
}

\author{
Jang Soo Kim $\|^{1}$ and Dennis Stanton 2 非 \\ ${ }^{1}$ Department of Mathematics, Sungkyunkwan University, Suwon, South Korea \\ ${ }^{2}$ School of Mathematics, University of Minnesota, Minneapolis, MN 55455
}

\begin{abstract}
A $q$-integral over an order polytope coming from a poset is interpreted as a generating function of linear extensions of the poset. As an application, the $q$-beta integral and a $q$-analog of Dirichlet's integral are computed. A combinatorial interpretation of a $q$-Selberg integral is also obtained.

Résumé. Une $q$-intégrale sur un polytope provenant d'un poset est interprété comme une série génératrice d'extensions linéaires de la poset. En application, l'intégrale $q$-bêta et un $q$-analogue de l'intégrale de Dirichlet sont calculés. Une interprétation combinatoire de une intégrale $q$-Selberg est également obtenue.
\end{abstract}

Keywords. $q$-integral, order polytope, $q$-Selberg integral

\section{Introduction}

In this extended abstract we give a combinatorial interpretation of $q$-integrals over order polytopes. The motivation of this extended abstract is to generalize Stanley's combinatorial interpretation of the Selberg integral.

The Selberg integral is the following integral first evaluated by Selberg [8] in 1944:

$$
\begin{aligned}
S_{n}(\alpha, \beta, \gamma) & =\int_{0}^{1} \cdots \int_{0}^{1} \prod_{i=1}^{n} x_{i}^{\alpha-1}\left(1-x_{i}\right)^{\beta-1} \prod_{1 \leq i<j \leq n}\left|x_{i}-x_{j}\right|^{2 \gamma} d x_{1} \cdots d x_{n} \\
& =\prod_{j=1}^{n} \frac{\Gamma(\alpha+(j-1) \gamma) \Gamma(\beta+(j-1) \gamma) \Gamma(1+j \gamma)}{\Gamma(\alpha+\beta+(n+j-2) \gamma) \Gamma(1+\gamma)}
\end{aligned}
$$

where $n$ is a positive integer and $\alpha, \beta, \gamma$ are complex numbers such that $\operatorname{Re}(\alpha)>0, \operatorname{Re}(\beta)>0$, and $\operatorname{Re}(\gamma)>-\min \{1 / n, \operatorname{Re}(\alpha) /(n-1), \operatorname{Re}(\beta) /(n-1)\}$. Stanley [9, Exercise $1.10(\mathrm{~b})]$ found a combinatorial interpretation of the above integral when $\alpha-1, \beta-1$ and $2 \gamma$ are nonnegative integers in terms of permutations. His idea is to interprete the integral as the probability that a random permutation satisfies

\footnotetext{
${ }^{\dagger}$ The first author was partially supported by Basic Science Research Program through the National Research Foundation of Korea (NRF) funded by the Ministry of Education (NRF-2013R1A1A2061006).

${ }^{\ddagger}$ The second author was supported by NSF grant DMS-1148634.
}

1365-8050 @ 2016 Discrete Mathematics and Theoretical Computer Science (DMTCS), Nancy, France 
certain properties. This idea uses the fact that a real number $x \in(0,1)$ can be understood as the probability that a random number selected from $(0,1)$ lies on an interval of length $x$ is equal to $x$. Generalizing this fact to $q$-integrals is not obvious. We instead consider a different approach by interpreting $q$-integrals as generating functions in $q$.

Throughout this extended abstract we assume $0<q<1$. We will use the following notation for $q$-series:

$$
[n]_{q}=\frac{1-q^{n}}{1-q}, \quad[n]_{q} !=[1]_{q}[2]_{q} \cdots[n]_{q}, \quad(a ; q)_{n}=(1-a)(1-a q) \cdots\left(1-a q^{n-1}\right) .
$$

We also use the notation $[n]:=\{1,2, \ldots, n\}$. We denote by $\mathfrak{S}_{n}$ the set of permutations on $[n]$.

In order to state our main results, we need several definitions. First, recall that the $q$-integral of a function $f$ over $(a, b)$ is

$$
\int_{a}^{b} f(x) d_{q} x=(1-q) \sum_{i=0}^{\infty}\left(f\left(b q^{i}\right) b q^{i}-f\left(a q^{i}\right) a q^{i}\right) .
$$

Note that the limit as $q \rightarrow 1$, the $q$-integral becomes the usual integral. It is well known that

$$
\int_{a}^{b} x^{n} d_{q} x=\frac{b^{n+1}-a^{n-1}}{[n+1]_{q}}
$$

For a permutation $\pi=\pi_{1} \ldots \pi_{n} \in \mathfrak{S}_{n}$ we will denote the region

$$
\left\{\left(x_{1}, \ldots, x_{n}\right): a \leq x_{\pi_{1}} \leq \cdots \leq x_{\pi_{n}} \leq b\right\}
$$

by $a \leq x_{\pi_{1}} \leq \cdots \leq x_{\pi_{n}} \leq b$. The $q$-integral over this region is defined as follows.

Definition 1.1. For a permutation $\pi=\pi_{1} \ldots \pi_{n} \in \mathfrak{S}_{n}$, we define

$$
\int_{a \leq x_{\pi_{1}} \leq \cdots \leq x_{\pi_{n}} \leq b} f\left(x_{1}, \ldots, x_{n}\right) d_{q} x_{1} \cdots d_{q} x_{n}=\int_{s_{n}}^{t_{n}} \cdots \int_{s_{1}}^{t_{1}} f\left(x_{1}, \ldots, x_{n}\right) d_{q} x_{1} \cdots d_{q} x_{n},
$$

where

$$
\begin{aligned}
s_{i} & =s_{i}\left(x_{i+1}, \ldots, x_{n}\right)=\max \left(\left\{x_{j}: j>i, \pi_{j}<\pi_{i}\right\} \cup\{a\}\right), \\
t_{i} & =t_{i}\left(x_{i+1}, \ldots, x_{n}\right)=\min \left(\left\{x_{j}: j>i, \pi_{j}>\pi_{i}\right\} \cup\{b\}\right) .
\end{aligned}
$$

For example,

$$
\begin{aligned}
\int_{a \leq x_{3} \leq x_{1} \leq x_{5} \leq x_{2} \leq x_{4} \leq x_{6} \leq b} f\left(x_{1}, \ldots, x_{6}\right) & d_{q} x_{1} \cdots d_{q} x_{6} \\
& =\int_{a}^{b} \int_{a}^{x_{6}} \int_{x_{5}}^{x_{6}} \int_{a}^{x_{5}} \int_{x_{5}}^{x_{4}} \int_{x_{3}}^{x_{5}} f\left(x_{1}, \ldots, x_{6}\right) d_{q} x_{1} \cdots d_{q} x_{6} .
\end{aligned}
$$

Note that since $s_{i}$ and $t_{i}$ are constants when $x_{i+1}, \ldots, x_{n}$ and $q$ are fixed, the above definition makes sense as a $q$-integral. 
Let $P$ be a poset on a set $\left\{x_{1}, x_{2}, \ldots, x_{n}\right\}$. By abuse of notation, we will consider $x_{i}$ as an element of $P$ and also as a variable. For $i \in[n]$, we denote by $P_{i}$ the subposet of $P$ consisting of $x_{i}, x_{i+1}, \ldots, x_{n}$. Let $\mathcal{O}_{a}^{b}(P)$ be the polytope

$$
\mathcal{O}_{a}^{b}(P)=\left\{\left(x_{1}, \ldots, x_{n}\right) \in[a, b]^{n}: x_{i} \leq x_{j} \text { if } x_{i} \leq_{P} x_{j}\right\}
$$

We will also use $\mathcal{O}(P)$ in place of $\mathcal{O}_{0}^{1}(P)$.

We define the $q$-integral over this polytope as follows.

Definition 1.2. The $q$-integral of $f\left(x_{1}, \ldots, x_{n}\right)$ over the polytope $\mathcal{O}_{a}^{b}(P)$ is defined by

$$
\int_{\mathcal{O}_{a}^{b}(P)} f\left(x_{1}, \ldots, x_{n}\right) d_{q} x_{1} \cdots d_{q} x_{n}=\int_{s_{n}}^{t_{n}} \int_{s_{n-1}}^{t_{n-1}} \cdots \int_{s_{1}}^{t_{1}} f\left(x_{1}, \ldots, x_{n}\right) d_{q} x_{1} \cdots d_{q} x_{n}
$$

where

$$
\begin{gathered}
s_{i}=s_{i}\left(x_{i+1}, \ldots, x_{n}\right)=\max \left(\{a\} \cup\left\{x_{k}: x_{k} \lessdot_{P_{i}} x_{i}\right\}\right), \\
t_{i}=t_{i}\left(x_{i+1}, \ldots, x_{n}\right)=\min \left(\{b\} \cup\left\{x_{k}: x_{i} \lessdot_{P_{i}} x_{k}\right\}\right) .
\end{gathered}
$$

By definition we have

$$
\lim _{q \rightarrow 1} \int_{\mathcal{O}_{a}^{b}(P)} f\left(x_{1}, \ldots, x_{n}\right) d_{q} x_{1} \cdots d_{q} x_{n}=\int_{\mathcal{O}_{a}^{b}(P)} f\left(x_{1}, \ldots, x_{n}\right) d x_{1} \cdots d x_{n}
$$

Note that if $P$ is the chain $x_{\pi_{1}}<x_{\pi_{2}}<\cdots<x_{\pi_{n}}$ for a permutation $\pi \in \mathfrak{S}_{n}$, then $\mathcal{O}_{a}^{b}(P)$ is the simplex $\left\{\left(x_{1}, \ldots, x_{n}\right): a \leq x_{\pi_{1}} \leq \cdots \leq x_{\pi_{n}} \leq b\right\}$ and

$$
\int_{\mathcal{O}_{a}^{b}(P)} f\left(x_{1}, \ldots, x_{n}\right) d_{q} x_{1} \cdots d_{q} x_{n}=\int_{a \leq x_{\pi_{1}} \leq \cdots \leq x_{\pi_{n}} \leq b} f\left(x_{1}, \ldots, x_{n}\right) d_{q} x_{1} \cdots d_{q} x_{n} .
$$

The remainder of this extended abstract is organised as follows. In Section 2 we will find a formula for the $q$-integral over a simplex. In Section 3 we show that the $q$-volume of the order polytope $\mathcal{O}(P)$ can be written as a generating function for linear extensions of the poset $P$. In Section 4 we find a relation between $q$-integrals over order polytopes $\mathcal{O}(P)$ and $\mathcal{O}(Q)$ for two posets $P$ and $Q$ such that $Q$ is obtained from $P$ by adding a chain. In Section 5 we show that the $q$-beta integral and a $q$-analog of Dirichlet's integral can be computed using our methods. We also discuss a connection with the general $q$-beta integral of Andrews and Askey [2]. In Section 6 we express a $q$-Selberg integral in terms of the $q$-volume of certain order polytope and find a combinatorial interpretation for it.

\section{$2 q$-integrals over simplices}

In this section we compute $q$-integrals of a multivariate function over the simplex $\left\{\left(x_{1}, \ldots, x_{n}\right): a \leq\right.$ $\left.x_{\pi_{1}} \leq \cdots \leq x_{\pi_{n}} \leq b\right\}$.

Let $\pi=\pi_{1} \pi_{2} \ldots \pi_{n} \in \mathfrak{S}_{n}$. An integer $i \in[n-1]$ is called a descent of $\pi$ if $\pi_{i}>\pi_{i+1}$. Let Des $(\pi)$ be the set of descents of $\pi$. We define $\operatorname{des}(\pi)$ and maj $(\pi)$ to be the number of descents of $\pi$ and the sum of descents of $\pi$, respectively. 
Lemma 2.1. For $\pi \in \mathfrak{S}_{n}$ and $r, s \in\{0,1,2, \ldots\} \cup\{\infty\}$, we have

$$
\begin{gathered}
\int_{q^{r} \leq x_{\pi_{1}} \leq \cdots \leq x_{\pi_{n}} \leq q^{s}} f\left(x_{\pi_{1}}, \ldots, x_{\pi_{n}}\right) d_{q} x_{1} \cdots d_{q} x_{n} \\
=(1-q)^{n} \sum_{\substack{r>i_{1} \geq \cdots \geq i_{n} \geq s \\
i_{j}>i_{j+1}}} \sum_{\substack{j \in \operatorname{Des}(\pi) \\
j \in \operatorname{los}}} f\left(q^{i_{1}}, \ldots, q^{i_{n}}\right) q^{i_{1}+\cdots+i_{n}} .
\end{gathered}
$$

The following theorem gives a formula for the volume of the simplex.

Theorem 2.2. For $\pi \in \mathfrak{S}_{n}$ and real numbers $a<b$, we have

$$
\int_{a \leq x_{\pi_{1}} \leq \cdots \leq x_{\pi_{n}} \leq b} d_{q} x_{1} \cdots d_{q} x_{n}=\frac{b^{n} q^{\operatorname{maj}(\pi)}}{[n]_{q} !}\left(a q^{-\operatorname{des}(\pi)} / b ; q\right)_{n} .
$$

When $a=0$ and $b=1$ in Theorem 2.2 we obtain the following corollary.

Corollary 2.3. For $\pi \in \mathfrak{S}_{n}$,

$$
\int_{0 \leq x_{\pi_{1}} \leq \cdots \leq x_{\pi_{n}} \leq 1} d_{q} x_{1} \cdots d_{q} x_{n}=\frac{q^{\mathrm{maj}(\pi)}}{[n]_{q} !} .
$$

\section{$3 q$-integrals over order polytopes}

In this section we consider $q$-integrals over order polytopes. We need some of the $P$-partition theory in [9. Chapter 3].

Let $\pi \in \mathfrak{S}_{n}$. A function $f:[n] \rightarrow \mathbb{N}$ is called $\pi$-compatible if $f\left(\pi_{1}\right) \geq f\left(\pi_{2}\right) \geq \cdots \geq f\left(\pi_{n}\right)$ and $f\left(\pi_{i}\right)>f\left(\pi_{i+1}\right)$ for all $i \in \operatorname{Des}(\pi)$. Then for any function $f:[n] \rightarrow \mathbb{N}$, there is a unique $\pi \in \mathfrak{S}_{n}$ for which $f$ is $\pi$-compatible.

Let $P$ be a poset with $n$ elements. A labeling of $P$ is a bijection $\omega: P \rightarrow[n]$. A $(P, \omega)$-partition is a function $\sigma: P \rightarrow \mathbb{N}$ such that

- $\sigma(x) \geq \sigma(y)$ if $x \leq_{P} y$,

- $\sigma(x)>\sigma(y)$ if $x \leq_{P} y$ and $\omega(x)>\omega(y)$.

A linear extension of $P$ is an arrangement $\left(t_{1}, t_{2}, \ldots, t_{n}\right)$ of the elements in $P$ such that if $t_{i}<_{P} t_{j}$ then $i<j$. The Jordan-Hölder set $\mathcal{L}(P, \omega)$ is the set of permutations of the form $\omega\left(t_{1}\right) \omega\left(t_{2}\right) \cdots \omega\left(t_{n}\right)$ for some linear extension $\left(t_{1}, t_{2}, \ldots, t_{n}\right)$ of $P$.

Let $\mathcal{A}(P, \omega)$ denote the set of $(P, \omega)$-partitions. For a permutation $\pi \in \mathfrak{S}_{n}$, we denote by $S_{\pi}(P, \omega)$ the set of functions $\sigma: P \rightarrow \mathbb{N}$ such that $\sigma \circ \omega^{-1}$ is $\pi$-compatible. Notice that in the definition of $S_{\pi}(P, \omega)$ we only need the underlying set of $P$. Thus we can consider $S_{\pi}(P, \omega)$ when $P$ is a set with $n$ elements and $\omega: P \rightarrow[n]$ is a bijection. We will use the following facts [9, Lemma 3.15.3, Theorem 3.15.7]:

$$
\mathcal{A}(P, \omega)=\bigcup_{\pi \in \mathcal{L}(P, \omega)} S_{\pi}(P, \omega)
$$




$$
\sum_{\sigma \in \mathcal{A}(P, \omega)} q^{|\sigma|}=\frac{\sum_{\pi \in \mathcal{L}(P, \omega)} q^{\operatorname{maj}(\pi)}}{(q ; q)_{n}}
$$

Lemma 2.1 can be restated as follows.

Proposition 3.1. Let $P=\left\{x_{1}, \ldots, x_{n}\right\}$ with a bijection $\omega: P \rightarrow[n]$ defined by $\omega\left(x_{i}\right)=i$. Then for integers $r>s \geq 0$ and a permutation $\pi \in \mathfrak{S}_{n}$, we have

$$
\int_{q^{r} \leq x_{\pi_{1}} \leq \cdots \leq x_{\pi_{n}} \leq q^{s}} f\left(x_{1}, \ldots, x_{n}\right) d_{q} x_{1} \cdots d_{q} x_{n}=(1-q)^{n} \sum_{\substack{\sigma \in S_{\pi}(P, \omega) \\ r>\max (\sigma), \min (\sigma) \geq s}} f\left(q^{\sigma\left(x_{1}\right)}, \ldots, q^{\sigma\left(x_{n}\right)}\right) q^{|\sigma|}
$$

In particular, when $r \rightarrow \infty$ and $s=0$, we have

$$
\int_{0 \leq x_{\pi_{1}} \leq \cdots \leq x_{\pi_{n}} \leq 1} f\left(x_{1}, \ldots, x_{n}\right) d_{q} x_{1} \cdots d_{q} x_{n}=(1-q)^{n} \sum_{\sigma \in S_{\pi}(P, \omega)} f\left(q^{\sigma\left(x_{1}\right)}, \ldots, q^{\sigma\left(x_{n}\right)}\right) q^{|\sigma|} .
$$

Proposition 3.2. Let $P$ be a poset on $\left\{x_{1}, \ldots, x_{n}\right\}$ with labeling $\omega: P \rightarrow[n]$ given by $\omega\left(x_{i}\right)=i$ for all $i \in[n]$. Then for real numbers $a<b$, we have

$$
\int_{\mathcal{O}_{a}^{b}(P)} f\left(x_{1}, \ldots, x_{n}\right) d_{q} x_{1} \cdots d_{q} x_{n}=\sum_{\pi \in \mathcal{L}(P, \omega)} \int_{a \leq x_{\pi_{1}} \leq \cdots \leq x_{\pi_{n}} \leq b} f\left(x_{1}, \ldots, x_{n}\right) d_{q} x_{1} \cdots d_{q} x_{n} .
$$

Theorem 3.3. Let $P$ be a poset on $\left\{x_{1}, \ldots, x_{n}\right\}$ with labeling $\omega: P \rightarrow[n]$ given by $\omega\left(x_{i}\right)=i$ for all $i \in[n]$. Then, we have

$$
\int_{\mathcal{O}(P)} f\left(x_{1}, \ldots, x_{n}\right) d_{q} x_{1} \cdots d_{q} x_{n}=(1-q)^{n} \sum_{\sigma \in \mathcal{A}(P, \omega)} f\left(q^{\sigma\left(x_{1}\right)}, \ldots, q^{\sigma\left(x_{n}\right)}\right) q^{|\sigma|}
$$

Moreover, if $Q=\mathcal{O}(P) \cap\left(\left[q^{r_{1}}, q^{s_{1}}\right] \times \cdots \times\left[q^{r_{n}}, q^{s_{n}}\right]\right)$ for $r_{i}, s_{i} \in\{0,1,2, \ldots\} \cup\{\infty\}$, then

$$
\int_{Q} f\left(x_{1}, \ldots, x_{n}\right) d_{q} x_{1} \cdots d_{q} x_{n}=(1-q)^{n} \sum_{\sigma} f\left(q^{\sigma\left(x_{1}\right)}, \ldots, q^{\sigma\left(x_{n}\right)}\right) q^{|\sigma|}
$$

where the sum is over all $\sigma \in \mathcal{A}(P, \omega)$ with $s_{i} \leq \sigma\left(x_{i}\right)<r_{i}$ for every $i \in\{1,2, \ldots, n\}$.

Thus, when $f\left(x_{1}, x_{2}, \ldots, x_{n}\right)=1$ in Theorem 3.3 , we obtain the following corollary.

Corollary 3.4. Let $P$ be a poset on $\left\{x_{1}, \ldots, x_{n}\right\}$ with labeling $\omega: P \rightarrow[n]$ given by $\omega\left(x_{i}\right)=i$ for all $i \in[n]$. Then

$$
\int_{\mathcal{O}(P)} d_{q} x_{1} \cdots d_{q} x_{n}=\frac{1}{[n]_{q} !} \sum_{\pi \in \mathcal{L}(P, \omega)} q^{\operatorname{maj}(\pi)}
$$




\section{Changing posets}

In this section we consider two posets $P$ and $Q$ where $Q$ is obtained from $P$ by adding a chain. The results in this section will be used for the next two sections.

Lemma 4.1. Let $\rho \in S_{m}$. Let $P$ be a poset on $\left\{x_{1}, \ldots, x_{n}\right\}$ with $x_{s} \leq_{P} x_{t}$. Define $Q$ to be the poset on $\left\{x_{1}, \ldots, x_{n}, y_{1}, y_{2}, \ldots, y_{m}\right\}$ with relations $x_{i} \leq_{Q} x_{j}$ if and only if $x_{i} \leq_{P} x_{j}$, and $x_{s} \leq_{Q} y_{\rho_{1}} \leq_{Q}$ $\cdots \leq_{Q} y_{\rho_{m}} \leq_{Q} x_{t}$. Then, we have

$$
\begin{gathered}
\int_{\mathcal{O}(P)} q^{\operatorname{maj}(\rho)} x_{t}^{m}\left(q^{-\operatorname{des}(\rho)} x_{s} / x_{t} ; q\right)_{m} f\left(x_{1}, \ldots, x_{n}\right) d_{q} x_{1} \cdots d_{q} x_{n} \\
=[m]_{q} ! \int_{\mathcal{O}(Q)} f\left(x_{1}, \ldots, x_{n}\right) d_{q} y_{1} \cdots d_{q} y_{m} d_{q} x_{1} \cdots d_{q} x_{n} \\
\int_{\mathcal{O}(P)} q^{\operatorname{maj}(\rho)} x_{t}^{m}\left(q^{1-\operatorname{des}(\rho)} x_{s} / x_{t} ; q\right)_{m} f\left(x_{1}, \ldots, x_{n}\right) d_{q} x_{1} \cdots d_{q} x_{n} \\
=[m]_{q} ! \int_{\mathcal{O}(Q)} f\left(x_{1}, \ldots, x_{n}\right) d_{q} x_{1} \cdots d_{q} x_{s} d_{q} y_{1} \cdots d_{q} y_{m} d_{q} x_{s+1} \cdots d_{q} x_{n}
\end{gathered}
$$

Moreover, (9) holds if the order of integration is obtained from $d_{q} x_{1} \cdots d_{q} x_{n}$ by inserting $d_{q} y_{1} \cdots d_{q} y_{m}$ anywhere between $x_{s}$ and $x_{t}$.

Similarly we can prove the following lemma.

Lemma 4.2. Let $\rho \in S_{m}$ and $s, t \in[m]$. Let $P$ be a poset on $\left\{x_{1}, \ldots, x_{n}\right\}$. Define $Q_{1}$ to be the poset on $\left\{x_{1}, \ldots, x_{n}, y_{1}, y_{2}, \ldots, y_{m}\right\}$ with relations $x_{i} \leq_{Q_{1}} x_{j}$ if and only if $x_{i} \leq_{P} x_{j}$, and $x_{s} \leq_{Q_{1}} y_{\rho_{1}} \leq_{Q_{1}}$ $\cdots \leq_{Q_{1}} y_{\rho_{m}}$. Define $Q_{2}$ to be the poset on $\left\{x_{1}, \ldots, x_{n}, y_{1}, y_{2}, \ldots, y_{m}\right\}$ with relations $x_{i} \leq_{Q_{2}} x_{j}$ if and only if $x_{i} \leq_{P} x_{j}$, and $y_{\rho_{1}} \leq_{Q_{2}} \cdots \leq_{Q_{2}} y_{\rho_{m}} \leq_{Q_{2}} x_{t}$. Then, we have

$$
\begin{gathered}
\int_{\mathcal{O}(P)} q^{\operatorname{maj}(\rho)}\left(q^{1-\operatorname{des}(\rho)} x_{s} ; q\right)_{m} f\left(x_{1}, \ldots, x_{n}\right) d_{q} x_{1} \cdots d_{q} x_{n} \\
=[m]_{q} ! \int_{\mathcal{O}\left(Q_{1}\right)} f\left(x_{1}, \ldots, x_{n}\right) d_{q} x_{1} \cdots d_{q} x_{n} d_{q} y_{1} \cdots d_{q} y_{m} \\
\int_{\mathcal{O}(P)} q^{\operatorname{maj}(\rho)} x_{t}^{m} f\left(x_{1}, \ldots, x_{n}\right) d_{q} x_{1} \cdots d_{q} x_{n} \\
=[m]_{q} ! \int_{\mathcal{O}\left(Q_{2}\right)} f\left(x_{1}, \ldots, x_{n}\right) d_{q} y_{1} \cdots d_{q} y_{m} d_{q} x_{1} \cdots d_{q} x_{n}
\end{gathered}
$$

\section{Examples}

In this section we will compute the $q$-beta integral and a $q$-analog of Dirichlet's integral using our results. We will then find a connection with the general $q$-beta integral due to Andrews and Askey [2]. 


\subsection{The q-beta integral}

The following is the well known integral called the $q$-beta integral. We can prove this using our methods.

Corollary 5.1. We have

$$
\int_{0}^{1} x^{n}(x q ; q)_{m} d_{q} x=\frac{[n]_{q} ![m]_{q} !}{[n+m+1]_{q} !} .
$$

Proof. By (10) and (11) we have

$$
\int_{0}^{1} x^{n}(x q ; q)_{m} d_{q} x=[n]_{q} ![m]_{q} ! \int_{0 \leq y_{1} \leq \cdots \leq y_{n} \leq x \leq z_{1} \leq \cdots \leq z_{m} \leq 1} d_{q} y_{1} \cdots d_{q} y_{n} d_{q} x d_{q} z_{1} \cdots d_{q} z_{m} .
$$

By Corollary 2.3 , we get the $q$-beta integral formula.

Corollary 5.2. Let $\pi$ be a permutation on $[n]$. Let $T$ be the poset obtained from the chain $\left\{\pi_{1}<\pi_{2}<\right.$ $\left.\cdots<\pi_{n}\right\}$ by adding $k_{i}$ elements covered by $\pi_{i}$. Then $T$ becomes a tree and for each element $v$ we define the hook length $h_{v}$ of $v$ to be the number of elements $u$ with $u \leq_{T} v$. Let maj $(T)=\sum_{i \in \operatorname{Des}(\pi)} h_{\pi_{i}}$. Then

$$
\int_{0 \leq x_{\pi_{1}} \leq \cdots \leq x_{\pi_{n}} \leq 1} x_{1}^{k_{1}} \cdots x_{n}^{k_{n}} d_{q} x_{1} \cdots d_{q} x_{n}=\frac{q^{\operatorname{maj}(T)}}{\prod_{v \in T}\left[h_{v}\right]_{q}} .
$$

Proof. This can be shown by applying (11) to each factor $x_{i}^{k_{i}}$.

\subsection{A q-analog of Dirichlet integral}

We now consider the simplex

$$
\Omega_{n}=\left\{\left(x_{1}, \ldots, x_{n}\right) \in[0,1]^{n}: x_{1}+\cdots+x_{n} \leq 1\right\} .
$$

Dirichlet's integral is the following, see [1, Theorem 1.8.1]:

$$
\int_{\Omega_{n}} x_{1}^{\alpha_{1}-1} \cdots x_{n}^{\alpha_{n}-1} d x_{1} \cdots d x_{n}=\frac{\Gamma\left(\alpha_{1}\right) \ldots \Gamma\left(\alpha_{n}\right)}{\Gamma\left(1+\alpha_{1}+\cdots+\alpha_{n}\right)} .
$$

By introducing new variables $y_{i}=x_{1}+\cdots+x_{i}$ and integers $k_{i}=\alpha_{i}+1$, we get an equivalent version of (12)

$$
\int_{0 \leq y_{1} \leq y_{2} \leq \cdots \leq y_{n} \leq 1} y_{1}^{k_{1}}\left(y_{2}-y_{1}\right)^{k_{2}} \cdots\left(y_{n}-y_{n-1}\right)^{k_{n}} d y_{1} \cdots d y_{n}=\frac{k_{1} ! \cdots k_{n} !}{\left(n+k_{1}+\cdots+k_{n}\right) !} .
$$

We obtain a $q$-analog of (13) as follows.

Corollary 5.3. For nonnegative integers $k_{1}, \ldots, k_{n}$, we have

$$
\int_{0 \leq y_{1} \leq y_{2} \leq \cdots \leq y_{n} \leq 1} y_{1}^{k_{1}}\left(q y_{1} / y_{2} ; q\right)_{k_{2}} y_{2}^{k_{2}} \cdots\left(q y_{n-1} / y_{n} ; q\right)_{k_{n}} y_{n}^{k_{n}} d_{q} y_{1} \cdots d_{q} y_{n}=\frac{\left[k_{1}\right]_{q} ! \cdots\left[k_{n}\right]_{q} !}{\left[n+k_{1}+\cdots+k_{n}\right]_{q} !} \text {. }
$$


Proof. By applying (11) to the factor $y_{1}^{k_{1}}$ and (9) to the factors $\left(q y_{i-1} / y_{i} ; q\right)_{k_{i}} y_{i}^{k_{i}}$ for $2 \leq i \leq n$, the left hand side is equal to

$$
\left[k_{1}\right]_{q} !\left[k_{2}\right]_{q} ! \cdots\left[k_{n}\right]_{q} ! \int_{Q} d_{q} x_{1,1} \cdots d_{q} x_{1, k_{1}} d_{q} y_{1} d_{q} x_{2,1} \cdots d_{q} x_{2, k_{2}} d_{q} y_{2} \cdots d_{q} x_{n, 1} \cdots d_{q} x_{n, k_{n}} d_{q} y_{n},
$$

where

$Q=\left\{0 \leq x_{1,1} \leq \cdots \leq x_{1, k_{1}} \leq y_{1} \leq x_{2,1} \leq \cdots \leq x_{2, k_{2}} \leq y_{2} \leq \cdots \leq x_{n, 1} \leq \cdots \leq x_{n, k_{n}} \leq y_{n} \leq 1\right\}$.

By Corollary 2.3 we obtain the right hand side.

\subsection{The general q-beta integral of Andrews and Askey}

In this subsection, we will show that Theorem 2.2 is related to the following result of Andrews and Askey [2] on a generalization of the $q$-beta integral: for $|q|<1$,

$$
\int_{a}^{b} \frac{(q x / a ; q)_{\infty}(q x / b ; q)_{\infty}}{(A x / a ; q)_{\infty}(B x / b ; q)_{\infty}} d_{q} x=\frac{(1-q)(q ; q)_{\infty}(A B ; q)_{\infty} a b(a / b ; q)_{\infty}(b / a ; q)_{\infty}}{(A ; q)_{\infty}(B ; q)_{\infty}(a-b)(B a / b ; q)_{\infty}(A b / a ; q)_{\infty}} .
$$

Let $\pi \in \mathfrak{S}_{n}$. We will compute the integral in Theorem 2.2 in a different way. First we decompose $\pi$ into $\pi=\sigma n \tau$ using the largest integer $n$. Suppose that $\sigma$ and $\tau$ have $r$ and $s$ letters respectively and $\operatorname{des}(\sigma)=k_{1}, \operatorname{des}(\tau)=k_{2}$. Then $n=r+s+1$ and $k=k_{1}+k_{2}+1$ if $k_{2} \geq 1$ and $k=k_{1}$ if $k_{2}=0$. The integral in Theorem 2.2 can be written as

$$
\int_{a}^{b}\left(\int_{a \leq x_{\sigma_{1}} \leq \cdots \leq x_{\sigma_{r}} \leq x_{n}} d_{q} y_{1} \cdots d_{q} y_{r} \int_{x_{n} \leq x_{\tau_{1}} \leq \cdots \leq x_{\tau_{s}} \leq b} d_{q} z_{1} \cdots d_{q} z_{s}\right) d_{q} x_{n},
$$

where $y_{1}, \ldots, y_{r}$ and $z_{1}, \ldots, z_{s}$ are obtained by rearranging $x_{\sigma_{1}}, \ldots, x_{\sigma_{r}}$ and $x_{\tau_{1}}, \ldots, x_{\tau_{s}}$ respectively so that subscripts are increasing. By applying Theorem 2.2 to the two inside integrals, the above is equal to

$$
\int_{a}^{b} \frac{x^{r} q^{\operatorname{maj}(\sigma)}}{[r]_{q} !}\left(a q^{-k_{1}} / x ; q\right)_{r} \frac{b^{s} q^{\operatorname{maj}(\tau)}}{[s]_{q} !}\left(x q^{-k_{2}} / b ; q\right)_{s} d_{q} x .
$$

Note that $\operatorname{maj}(\pi)=\operatorname{maj}(\sigma)+\operatorname{maj}(\tau)+(r+1)\left(k_{2}+1\right)$ if $s \geq 1$ and $\operatorname{maj}(\pi)=\operatorname{maj}(\sigma)$ if $s=0$. In either case we can write $\operatorname{maj}(\pi)=\operatorname{maj}(\sigma)+\operatorname{maj}(\tau)+(r+1)\left(k-k_{1}\right)$. Since (15) is equal to the integral in Theorem 2.2 we obtain the following.

Proposition 5.4. Let $n, r, s, k_{1}, k_{2}$ be nonnegative integers such that $n=r+s+1, k_{1} \leq r, k_{2} \leq s$, and $k=k_{1}+k_{2}+1$ if $s \geq 1$ and $k=k_{1}$ if $s=0$. Then

$$
\int_{a}^{b} x^{r}\left(a q^{-k_{1}} / x ; q\right)_{r}\left(x q^{-k_{2}} / b ; q\right)_{s} d_{q} x=\frac{[r]_{q} ![s]_{q} !}{[n]_{q} !} b^{r+1} q^{\left(k-k_{1}\right)(r+1)}\left(a q^{-k} / b ; q\right)_{n} .
$$

We now consider the case $s \geq 1$ in Proposition 5.4 so that $k=k_{1}+k_{2}+1$. One can rewrite the integral in Proposition 5.4 as

$$
\begin{aligned}
&(-1)^{r} a^{r} q^{\left(\begin{array}{r}
r \\
2
\end{array}\right)-k_{1} r} \int_{a}^{b} \frac{\left(x q^{1-r+k_{1}} / a ; q\right)_{\infty}\left(x q^{-k_{2}} / b ; q\right)_{\infty}}{\left(x q^{k_{1}+1} / a ; q\right)_{\infty}\left(x q^{s-k_{2}} / b ; q\right)_{\infty}} d_{q} x \\
& \quad=(-1)^{r} a^{r} q^{\left(\begin{array}{r}
r \\
2
\end{array}\right)-k_{1} r} \int_{a q^{r-k_{1}}}^{b q^{k_{2}+1}} \frac{\left(x q^{1-r+k_{1}} / a ; q\right)_{\infty}\left(x q^{-k_{2}} / b ; q\right)_{\infty}}{\left(x q^{k_{1}+1} / a ; q\right)_{\infty}\left(x q^{s-k_{2}} / b ; q\right)_{\infty}} d_{q} x
\end{aligned}
$$


where the equality follows from the fact that the integrand is 0 if $x=b q^{j}$ for $0 \leq j \leq k_{2}$ and $x=a q^{j}$ for $0 \leq j \leq r-k_{1}-1$. Thus Proposition 5.4 is equivalent to

$$
\int_{a q^{r-k_{1}}}^{b q^{k_{2}+1}} \frac{\left(x q^{1-r+k_{1}} / a ; q\right)_{\infty}\left(x q^{-k_{2}} / b ; q\right)_{\infty}}{\left(x q^{k_{1}+1} / a ; q\right)_{\infty}\left(x q^{s-k_{2}} / b ; q\right)_{\infty}} d_{q} x=\frac{(-1)^{r} b^{r+1}[r]_{q} ![s]_{q} ! q^{k r+k-k_{1}-\left(\begin{array}{c}
r \\
2
\end{array}\right)\left(a q^{-k} / b ; q\right)_{n}}}{a^{r}[n]_{q} !} .
$$

One can check that the above equation is the special case of (14) with substitution

$$
(a, b, A, B) \mapsto\left(a q^{r-k_{1}}, b q^{k_{2}+1}, q^{r+1}, q^{s+1}\right) .
$$

\section{$6 \quad q$-Selberg integrals}

In this section we will find a combinatorial interpretation for a $q$-Selberg integral.

For a set of variables $x=\left(x_{1}, \ldots, x_{n}\right)$ we denote

$$
\Delta(x)=\prod_{1 \leq i<j \leq n}\left(x_{i}-x_{j}\right) .
$$

There are many generalizations of the Selberg integral, see [4]. In this section we consider the following two $q$-Selberg integrals:

$$
\begin{aligned}
& \operatorname{AS}_{n}(\alpha, \beta, m)=\int_{0}^{1} \cdots \int_{0}^{1} \prod_{i=1}^{n} x_{i}^{\alpha-1}\left(q x_{i} ; q\right)_{\beta-1} \prod_{1 \leq i<j \leq n} x_{j}^{2 m}\left(q^{1-m} x_{i} / x_{j} ; q\right)_{2 m} d_{q} x_{1} \cdots d_{q} x_{n}, \\
& \operatorname{KS}_{n}(\alpha, \beta, m)=\int_{0}^{1} \cdots \int_{0}^{1} \prod_{i=1}^{n} x_{i}^{\alpha-1}\left(q x_{i} ; q\right)_{\beta-1} \Delta_{n, m}(x) d_{q} x_{1} \cdots d_{q} x_{n},
\end{aligned}
$$

where

$$
\Delta_{n, m}(x)=\prod_{1 \leq i<j \leq n} x_{j}^{m}\left(q^{1-m} x_{i} / x_{j} ; q\right)_{m} x_{j}^{m}\left(x_{i} / x_{j} ; q\right)_{m}=\Delta(x) \prod_{1 \leq i<j \leq n} x_{j}^{2 m-1}\left(q^{1-m} x_{i} / x_{j} ; q\right)_{2 m-1} .
$$

It is easy to check that $\Delta_{n, m}(x)$ is symmetric in the variables $x_{1}, x_{2}, \ldots, x_{n}$.

Askey [3] conjectured that

$$
\operatorname{AS}_{n}(\alpha, \beta, m)=q^{\alpha m\left(\begin{array}{c}
n \\
2
\end{array}\right)+2 m^{2}\left(\begin{array}{c}
n \\
3
\end{array}\right)} \prod_{j=1}^{n} \frac{\Gamma_{q}(\alpha+(j-1) m) \Gamma_{q}(\beta+(j-1) m) \Gamma_{q}(1+j m)}{\Gamma_{q}(\alpha+\beta+(n+j-2) m) \Gamma_{q}(1+m)},
$$

which has been proved by Habsieger [5] and Kadell [6] independently. Kadell [7, Eq. (4.11)] showed that

$$
\operatorname{AS}_{n}(\alpha, \beta, m)=\frac{[n]_{q^{m}} !}{n !} \mathrm{KS}_{n}(\alpha, \beta, m) .
$$

Since the integrand of $\operatorname{KS}_{n}(\alpha, \beta, m)$ is symmetric under any permutation of $x_{1}, \ldots, x_{n}$ and zero whenever $x_{i}=x_{j}$, we have

$$
\mathrm{KS}_{n}(\alpha, \beta, m)=n ! \int_{0 \leq x_{1} \leq \cdots \leq x_{n} \leq 1} \prod_{i=1}^{n} x_{i}^{\alpha-1}\left(q x_{i} ; q\right)_{\beta-1} \Delta_{n, m}(x) d_{q} x_{1} \cdots d_{q} x_{n}
$$


Thus we have

$$
\begin{aligned}
\operatorname{AS}_{n}(\alpha, \beta, m) & =\int_{0}^{1} \cdots \int_{0}^{1} \prod_{i=1}^{n} x_{i}^{\alpha-1}\left(q x_{i} ; q\right)_{\beta-1} \prod_{1 \leq i<j \leq n} x_{i}^{2 m}\left(q^{1-m} x_{j} / x_{i} ; q\right)_{2 m} d_{q} x_{1} \cdots d_{q} x_{n} \\
& =[n]_{q^{m}} ! \int_{0 \leq x_{1} \leq \cdots \leq x_{n} \leq 1} \prod_{i=1}^{n} x_{i}^{\alpha-1}\left(q x_{i} ; q\right)_{\beta-1} \Delta_{n, m}(x) d_{q} x_{1} \cdots d_{q} x_{n} .
\end{aligned}
$$

By (16) and [17], we have

$$
\begin{array}{r}
\int_{0 \leq x_{1} \leq \cdots \leq x_{n} \leq 1} \prod_{i=1}^{n} x_{i}^{\alpha-1}\left(q x_{i} ; q\right)_{\beta-1} \prod_{1 \leq i<j \leq n} x_{j}^{2 m-1}\left(q^{1-m} x_{i} / x_{j} ; q\right)_{2 m-1} \Delta(x) d_{q} x_{1} \cdots d_{q} x_{n} \\
=q^{\alpha m\left(\begin{array}{c}
n \\
2
\end{array}\right)+2 m^{2}\left(\begin{array}{c}
n \\
3
\end{array}\right)} \prod_{j=1}^{n} \frac{\Gamma_{q}(\alpha+(j-1) m) \Gamma_{q}(\beta+(j-1) m) \Gamma_{q}(j m)}{\Gamma_{q}(\alpha+\beta+(n+j-2) m) \Gamma_{q}(m)}
\end{array}
$$

We define the Selberg poset $P(n, r, s, m)$ to be the poset in which the elements are $x_{i}, y_{i}^{(a)}, z_{i}^{(b)}, w_{i, j}^{(k)}$ for $i, j \in[n], a \in[r], b \in[s], k \in[m]$ with $i \neq j$, and the covering relations are as follows:

- $x_{i}<w_{i, j}^{(1)}<\cdots<w_{i, j}^{(m)}<x_{j}$ for $1 \leq i<j \leq n$,

- $x_{i}<w_{j, i}^{(m)}<\cdots<w_{j, i}^{(1)}<x_{j}$ for $1 \leq i<j \leq n$,

- $y_{i}^{(1)}<\cdots<y_{i}^{(r)}<x_{i}<z_{i}^{(1)}<\cdots<z_{i}^{(s)}$ for $1 \leq i \leq n$.

For an example of $P(n, r, s, m)$, see Figure 1 .

The following theorem implies that the $q$-Selberg integral is the $q$-volume of an order polytope up to a certain factor.

Theorem 6.1. We have

$$
\begin{aligned}
& \int_{0 \leq x_{1} \leq \cdots \leq x_{n} \leq 1} \prod_{i=1}^{n} x_{i}^{r}\left(q x_{i} ; q\right)_{s} \prod_{1 \leq i<j \leq n} x_{j}^{m}\left(q^{1-m} x_{i} / x_{j} ; q\right)_{m} x_{j}^{m}\left(x_{i} / x_{j} ; q\right)_{m} d_{q} x_{1} \cdots d_{q} x_{n} \\
& =q^{-\left(\begin{array}{c}
m \\
2
\end{array}\right)\left(\begin{array}{c}
n \\
2
\end{array}\right)}\left([r]_{q} !\right)^{n}\left([s]_{q} !\right)^{n}\left([m]_{q} !\right)^{2\left(\begin{array}{c}
n \\
2
\end{array}\right)} \int_{\mathcal{O}(P(n, r, s, m))} d_{q} W d_{q} Y d_{q} X d_{q} Z,
\end{aligned}
$$

where the order of integration $d_{q} W d_{q} Y d_{q} X d_{q} Z$ is given by

$$
\left(\prod_{1 \leq i \neq j \leq n} d_{q} w_{i, j}^{(1)} \cdots d_{q} w_{i, j}^{(m)}\right)\left(\prod_{i=1}^{n} d_{q} y_{i}^{(1)} \cdots d_{q} y_{i}^{(r)}\right)\left(\prod_{i=1}^{n} d_{q} x_{i}\right)\left(\prod_{i=1}^{n} d_{q} z_{i}^{(1)} \cdots d_{q} z_{i}^{(s)}\right) .
$$

In the order of integration (19), the order of $d_{q} w_{i, j}^{(k)}$ and $d_{q} w_{i^{\prime}, j^{\prime}}^{(k)}$ for $(i, j) \neq\left(i^{\prime}, j^{\prime}\right)$ does not matter. The following corollary gives a combinatorial interpretation for the $q$-Selberg integral in terms of linear extensions. 


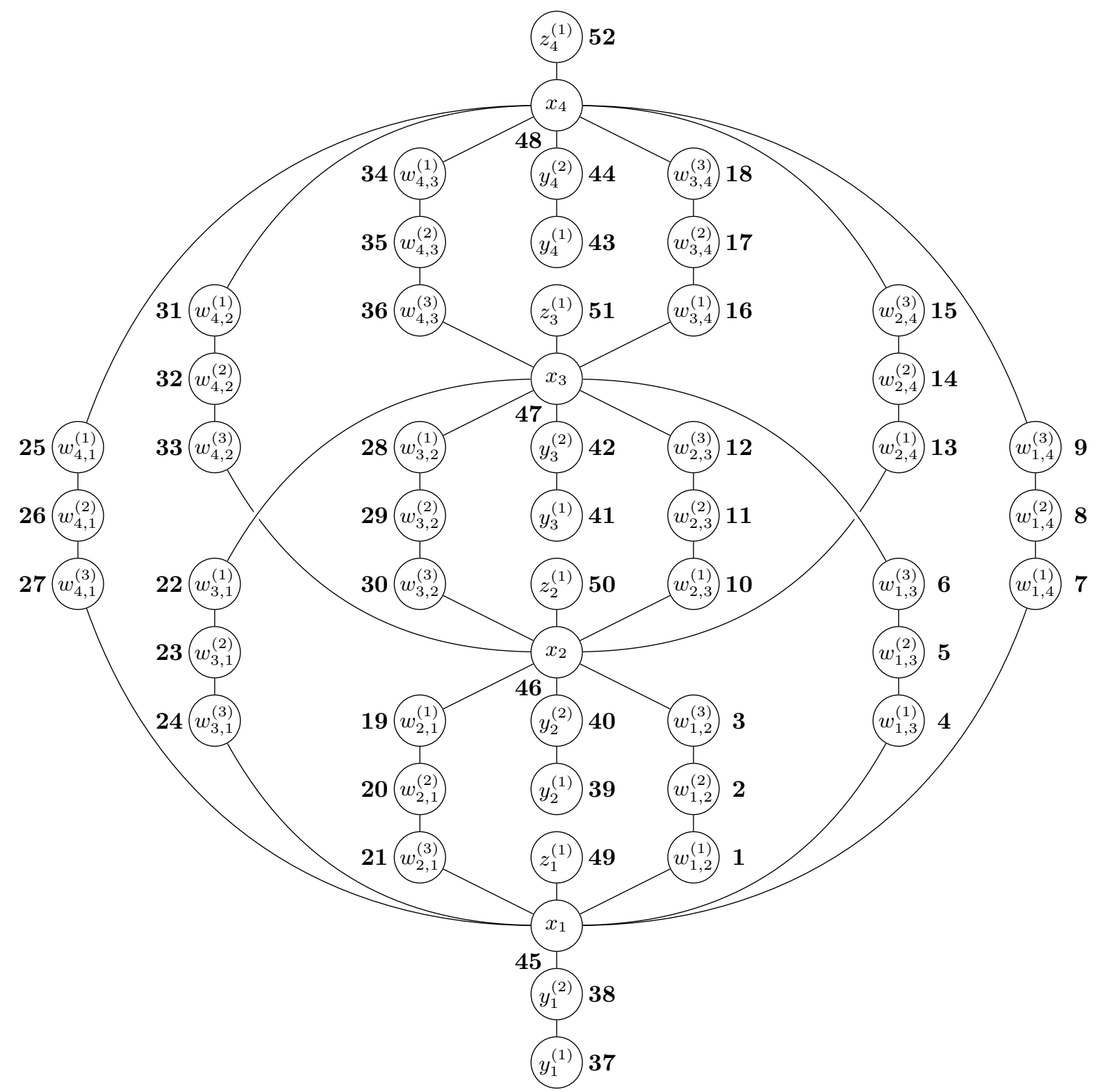

Fig. 1: The Selberg poset $P(n, r, s, m)$ for $n=4, r=2, s=1, m=3$ with labeling. 
Corollary 6.2. We have

$$
\begin{aligned}
\int_{0 \leq x_{1} \leq \cdots \leq x_{n} \leq 1} \prod_{i=1}^{n} x_{i}^{r}\left(q x_{i} ; q\right)_{s} & \prod_{1 \leq i<j \leq n} x_{j}^{m}\left(q^{1-m} x_{i} / x_{j} ; q\right)_{m} x_{j}^{m}\left(x_{i} / x_{j} ; q\right)_{m} d_{q} x_{1} \cdots d_{q} x_{n} \\
& =\frac{q^{-\left(\begin{array}{c}
m \\
2
\end{array}\right)\left(\begin{array}{c}
n \\
2
\end{array}\right)\left([r]_{q} !\right)^{n}\left([s]_{q} !\right)^{n}\left([m]_{q} !\right)^{2}\left(\begin{array}{c}
n \\
2
\end{array}\right)}}{[N]_{q} !} \sum_{\pi \in \mathcal{L}(P(n, r, s, m), \omega)} q^{\operatorname{maj}(\pi)}
\end{aligned}
$$

where

$$
N=n(r+s+1)+2 m\left(\begin{array}{l}
n \\
2
\end{array}\right)
$$

and, $\omega$ is the labeling of the Selberg poset $P(n, r, s, m)$ as shown in Figure 1 .

Corollary 6.3. We have

$$
\begin{aligned}
& \sum_{\pi \in \mathcal{L}(P(n, r, s, m), \omega)} q^{\operatorname{maj}(\pi)} \\
& =\frac{q^{\left(\begin{array}{c}
m \\
2
\end{array}\right)\left(\begin{array}{c}
n \\
2
\end{array}\right)+(r+1) m\left(\begin{array}{c}
n \\
2
\end{array}\right)+2 m^{2}\left(\begin{array}{c}
n \\
3
\end{array}\right)[N]_{q} !}}{\left([r]_{q} !\right)^{n}\left([s]_{q} !\right)^{n}\left([m]_{q} !\right)^{2\left(\begin{array}{c}
n \\
2
\end{array}\right)}} \prod_{j=1}^{n} \frac{[r+(j-1) m]_{q} ![s+(j-1) m]_{q} ![j m-1]_{q} !}{[r+s+1+(n+j-2) m]_{q} ![m-1]_{q} !},
\end{aligned}
$$

where $N$ and $\omega$ are the same as in Corollary 6.2

\section{References}

[1] G. E. Andrews, R. Askey, and R. Roy. Special functions, volume 71 of Encyclopedia of Mathematics and its Applications. Cambridge University Press, Cambridge, 1999.

[2] G. E. Andrews and R. Askey. Another $q$-extension of the beta function. Proc. Amer. Math. Soc., 81(1):97-100, 1981.

[3] R. Askey. Some basic hypergeometric extensions of integrals of Selberg and Andrews. SIAM J. Math. Anal., 11(6):938-951, 1980.

[4] P. J. Forrester and S. O. Warnaar. The importance of the Selberg integral. Bull. Amer. Math. Soc. (N.S.), 45(4):489-534, 2008.

[5] L. Habsieger. Une $q$-intégrale de Selberg et Askey. SIAM J. Math. Anal., 19(6):1475-1489, 1988.

[6] K. W. J. Kadell. A proof of Askey's conjectured $q$-analogue of Selberg's integral and a conjecture of Morris. SIAM J. Math. Anal., 19(4):969-986, 1988.

[7] K. W. J. Kadell. A proof of some $q$-analogues of Selberg's integral for $k=1$. SIAM J. Math. Anal., 19(4):944-968, 1988.

[8] A. Selberg. Remarks on a multiple integral. Norsk Mat. Tidsskr., 26:71-78, 1944.

[9] R. P. Stanley. Enumerative Combinatorics. Vol. 1, second ed. Cambridge University Press, New York/Cambridge, 2011. 